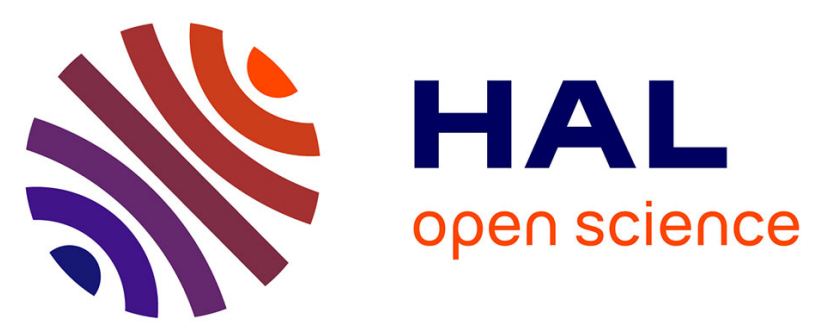

\title{
Molecular characterization of a Streptococcus gallolyticus genomic island encoding a pilus involved in endocarditis.
}

\author{
Camille C. Danne, José M J. M. Entenza, Adeline A. Mallet, Romain
}

Briandet, Michel M. Débarbouillé, Farida F. Nato, Philippe P. Glaser,

Grégory G. Jouvion, Philippe P. Moreillon, Patrick P. Trieu-Cuot, et al.

\section{To cite this version:}

Camille C. Danne, José M J. M. Entenza, Adeline A. Mallet, Romain Briandet, Michel M. Débarbouillé, et al.. Molecular characterization of a Streptococcus gallolyticus genomic island encoding a pilus involved in endocarditis.. Journal of Infectious Diseases, 2011, 204 (12), pp.1960-70. 10.1093/infdis/jir666 . hal-01000633

\section{HAL Id: hal-01000633 https://hal.science/hal-01000633}

Submitted on 5 Jun 2014

HAL is a multi-disciplinary open access archive for the deposit and dissemination of scientific research documents, whether they are published or not. The documents may come from teaching and research institutions in France or abroad, or from public or private research centers.
L'archive ouverte pluridisciplinaire HAL, est destinée au dépôt et à la diffusion de documents scientifiques de niveau recherche, publiés ou non, émanant des établissements d'enseignement et de recherche français ou étrangers, des laboratoires publics ou privés. 


\title{
Molecular Characterization of a Streptococcus gallolyticus Genomic Island Encoding a Pilus Involved in Endocarditis
}

\author{
Camille Danne, ${ }^{1,2,3}$ José M. Entenza, ${ }^{9}$ Adeline Mallet, ${ }^{4}$ Romain Briandet, ${ }^{8}$ Michel Débarbouillé, ${ }^{1,2}$ Farida Nato, ${ }^{5}$ \\ Philippe Glaser, ${ }^{6}$ Grégory Jouvion, ${ }^{7}$ Philippe Moreillon, ${ }^{9}$ Patrick Trieu-Cuot, ${ }^{1,2}$ and Shaynoor Dramsi ${ }^{1,2}$ \\ ${ }^{1}$ Unité de Biologie des Bactéries Pathogènes à Gram-Positif, ${ }^{2}$ Centre National de la Recherche Scientifique (CNRS) URA 2172, ${ }^{3}$ Université Paris 7-Denis \\ Diderot, ${ }^{4}$ Imagopole, Ultrastructural Microscopy Platform, ${ }^{5}$ Laboratoire de Production de Protéines Recombinantes et d'Anticorps, ${ }^{6}$ Laboratoire Evolution \\ et Génomique Bactériennes and CNRS URA 2171, and 7Unité Histopathologie Humaine et Modèles Animaux, Institut Pasteur, Paris, and ${ }^{8}$ nstitut \\ National de Recherche Agronomique, Micalis UMR 1319, Massy, France; and 9University of Lausanne, Department of Fundamental Microbiology, \\ Switzerland
}

Background. Streptococcus gallolyticus is a causative agent of infective endocarditis associated with colon cancer. Genome sequence of strain UCN34 revealed the existence of 3 pilus loci (pill, pil2, and pil3). Pili are long filamentous structures playing a key role as adhesive organelles in many pathogens. The pill locus encodes 2 LPXTG proteins (Gallo2178 and Gallo2179) and 1 sortase C (Gallo2177). Gallo2179 displaying a functional collagen-binding domain was referred to as the adhesin, whereas Gallo2178 was designated as the major pilin.

Methods. S. gallolyticus UCN34, Pill ${ }^{+}$and Pill ${ }^{-}$, expressing various levels of pill, and recombinant Lactococcus lactis strains, constitutively expressing pill, were studied. Polyclonal antibodies raised against the putative pilin subunits Gallo2178 and Gallo2179 were used in immunoblotting and immunogold electron microscopy. The role of pill was tested in a rat model of endocarditis.

Results. We showed that the pill locus (gallo2179-78-77) forms an operon differentially expressed among S. gallolyticus strains. Short pilus appendages were identified both on the surface of S. gallolyticus UCN34 and recombinant $L$. lactis-expressing pill. We demonstrated that Pill pilus is involved in binding to collagen, biofilm formation, and virulence in experimental endocarditis.

Conclusions. This study identifies Pill as the first virulence factor characterized in S. gallolyticus.

Streptococcus gallolyticus subsp gallolyticus (formerly known as Streptococcus bovis biotype I) is an increasing cause of infective endocarditis (IE). Asymptomatic carriage of $S$. gallolyticus is commonly observed in the gastrointestinal tract of birds, ruminants, and a small proportion of humans $(2.5 \%-15 \%)[1,2]$. Several studies have shown that endocarditis due to $S$. gallolyticus are frequently associated with colorectal carcinoma [1,3-6]. Whether the development of tumors is a cause or

Received 14 April 2011; accepted 16 July 2011; electronically published 31 October 2011.

Correspondence: Shaynoor Dramsi, PhD, Institut Pasteur, Unité de Biologie des Bactéries Pathogènes à Gram-Positif, CNRS URA 2172, 25, rue du Dr Roux, Paris, France 75015 (shaynoor.dramsi@pasteur.fr).

The Journal of Infectious Diseases 2011;204:1960-70

(C) The Author 2011. Published by Oxford University Press on behalf of the Infectious Diseases Society of America. All rights reserved. For Permissions, please e-mail: journals.permissions@oup.com

0022-1899 (print)/1537-6613 (online)/2011/20412-0020\$14.00

DOI: 10.1093/infdis/jir666 a consequence of $S$. gallolyticus infections remains to be investigated. To address this question, a better understanding of the pathophysiology of these diseases is required with a focus on the bacterial virulence factors responsible for the initiation of infections, that is, adhesion to host tissues.

The gallate-degrading strains of $S$. bovis group have been reassigned to a new species named $S$. gallolyticus sp [7-9]. Customarily, human isolates of S. bovis were classified into 3 biotypes designated as I, II/1, and II/2 that corresponds to $S$. gallolyticus subsp gallolyticus, the closely related subspecies pasteurianus, and the more distant subspecies infantarius, respectively. Finally, Streptococcus macedonicus is considered as a nonpathogenic S. gallolyticus subspecies. However, this taxonomic reclassification is still a matter of debate, and for simplification and readability these subspecies will be considered as species throughout this work. 
Although the proportion of IE due to S. gallolyticus has increased among streptococci, particularly in Southern Europe [10-12], its virulence and colonization factors remain largely unknown. Five serotypes have been described based on capsular typing in strains isolated from pigeons [13]. Electron microscopic studies of $S$. gallolyticus pigeon strains revealed the existence of filamentous structures known as fimbriae or pili [14]. It was hypothesized that these structures could play a role in virulence. Bacterial pathogens associated with IE possess surface adhesins belonging to MSCRAMMs (microbial surface components recognizing adhesive matrix molecules), which mediate attachment to cardiac vegetations and are involved in valve colonization and infection [15]. Previously characterized collagen-binding proteins include Cna of Staphylococcus aureus [16], Acm of Enterococcus faecium [17], Ace of E. faecalis [18, 19], and Acb from S. gallolyticus strain TX20005 [20]. Development of endocarditis is initiated by injury of the endothelium, which disrupts the normal valve structure and exposes underlying tissues, including extracellular matrix (ECM) material. Deposition of host proteins, such as fibrin and platelets, then leads to the formation of a sterile thrombotic vegetation that may become colonized by circulating bacteria [21].

S. gallolyticus isolates responsible for IE were shown to display heterogeneous profiles of adherence to ECM proteins [22-24]. We recently participated in the complete sequencing of S. gallolyticus strain UCN34, isolated from a human IE case associated with colorectal cancer [25]. In silico analyses enabled the identification of 19 putative cell wall-anchored proteins. We focused on 3 putative pilus loci, each encoding 1 sortase $\mathrm{C}$ and 2 LPXTG motif proteins, referred herein as pil1 (gallo2179-77), pil2 (gallo1570-68), and pil3 (gallo2040-38). It was recently reported that S. gallolyticus strain TX20005 (draft genome) also encodes 3 pilus loci [20]. Interestingly, only 2 of these loci are identical in both S. gallolyticus strains, namely pill (acbsbs7-srtC1 in TX20005) and pil3 (sbs15-sbs14-srtC2 in TX20005). Gram-positive pili were first observed in Corynebacterium renale by electron microscopy [26] and have now been characterized genetically and biochemically in many important pathogens, for example Streptococcus agalactiae [27-30]. These pili consist of covalently cross-linked subunit proteins and are anchored to the peptidoglycan (for reviews, see [31, 32]). Sortase-mediated pilus assembly was first demonstrated in Corynebacterium diphteriae [33, 34], and the current model for pilus biogenesis is as follows: the major subunit is assembled into the pilus by a cisencoded class $\mathrm{C}$ sortase that catalyzes the covalent attachment between the conserved lysyl residue of the pilin motif (WxxxVxVYPK) of one subunit and the conserved threonyl residue of the LPXTG motif of another subunit [35]. In addition, one or more accessory subunits could also be incorporated into the pilus backbone [36].

Here, we studied the pil1 pilus locus of S. gallolyticus UCN34, which is present in $90 \%$ of $S$. gallolyticus IE clinical isolates but absent in the closely related nonpathogenic species $S$. macedonicus (P. Glaser, unpublished data). Moreover, pil1 was predicted to encode a pilus made of 2 pilin subunits: the major pilin (Gallo2178) and the adhesin (Gallo2179), which displays a functional collagen-binding domain. We identified pilus appendages on the surface of S. gallolyticus and tested the role of Pill pilus in adhesion to ECM proteins, biofilm formation, and virulence in a rat model of endocarditis.

\section{MATERIALS AND METHODS}

\section{Bacterial Strains, Plasmids, and Growth Conditions}

Bacterial strains, plasmids, and primers are listed in Tables 1 and 2. S. gallolyticus were grown at $37^{\circ} \mathrm{C}$ in Todd-Hewitt broth, in standing filled flasks. L. lactis strain NZ9000 [46] was grown in M17 medium supplemented with $1 \%$ glucose. Heterologous expression of pil1 in L. lactis strain was realized as follows: an $\sim 5$-kilobase DNA fragment containing the 3 genes gallo2179-2178-2177 was amplified from UCN34 genomic DNA with primers pilUCN34-fwd and pilUCN34-rev (Table 2), digested by BamH1 and Nsil (New England Biolabs) and cloned into the high-copy-number erythromycin resistance shuttle vector pOri23 [38] digested by BamH1-Pst1. After ligation, the resulting plasmid pOri23 $\Omega$ pil1 was introduced into electrocompetent L. lactis NZ9000 cells. Unless otherwise specified, antibiotics were used at the following concentrations: for Escherichia coli, $150 \mu \mathrm{g} / \mathrm{mL}$ erythromycin; for S. gallolyticus, $10 \mu \mathrm{g} / \mathrm{mL}$ tetracycline; and for L. lactis, $5 \mu \mathrm{g} /$ $\mathrm{mL}$ erythromycin.

\section{Real-time Polymerase Chain Reaction}

Total RNA $(15 \mu \mathrm{g})$ were extracted and treated as described elsewhere [27]. Quantitative real-time polymerase chain reaction (PCR) analysis was performed as described elsewhere [27] with gene-specific primers (Table 2).

\section{Cell Wall Protein Extracts}

Bacteria were grown in Todd-Hewitt medium at $37^{\circ} \mathrm{C}$ and harvested for protein analysis during late exponential phase of culture. Cell wall extracts were prepared as described elsewhere [27].

\section{Expression and Purification of Recombinant 6xHis-Gallo2178 and 6xHis-Gallo2179}

DNA fragments internal to gallo2179 and gallo2178 were produced by PCR using genomic DNA of UCN34 as the template and the primers gallo2179-Nhe1 and gallo2179-BamH1, and gallo2178-Nhe1 and gallo2178-BamH1, respectively (Supplementary Table 1). These DNA fragments were digested with the appropriate enzymes (Nhe1 and Bam $\mathrm{H} 1$ ) and cloned into pET28-a $(+)$ (Novagen). The resulting plasmids were introduced into $E$. coli strain $\mathrm{DH} 5 \alpha$ for sequence analysis or BL21( $\lambda \mathrm{DE} 3)$ 
Table 1. Bacterial Strains and Plasmids Used in Current Study

\begin{tabular}{|c|c|c|c|}
\hline Bacterial Strains and Plasmids & Characterization & Antibiotic Resistance & References \\
\hline \multicolumn{4}{|l|}{ Escherichia coli } \\
\hline$D H 5 \alpha$ & 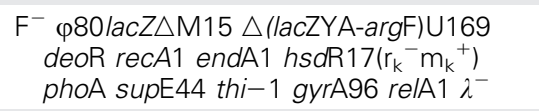 & Em & Invitrogen \\
\hline $\mathrm{BL21}(\lambda \mathrm{DE} 3)$ & 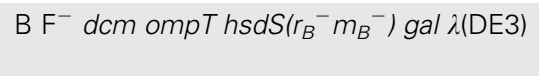 & & $\begin{array}{l}\text { Novagen, } \\
\text { R\&D Systems }\end{array}$ \\
\hline \multicolumn{4}{|l|}{$\begin{array}{l}\text { Streptococcus } \\
\text { gallolyticus }\end{array}$} \\
\hline UCN34 & $\begin{array}{l}\text { MLST 1, infectious endocarditis } \\
\text { and colon cancer }\end{array}$ & Tc & R. Leclercq, [1] \\
\hline Pil1 $^{+}$(NEM2470) & MLST 1, infectious endocarditis & Tc & $\begin{array}{l}\text { R. Leclercq, } \\
\text { Caen Hospital }\end{array}$ \\
\hline Pil1- (NEM2474) & MLST 25, infectious endocarditis & Tc & $\begin{array}{l}\text { R. Leclercq, } \\
\text { Caen Hospital }\end{array}$ \\
\hline \multicolumn{4}{|l|}{ Lactococcus lactis } \\
\hline NZ9000/pOri23 & $\begin{array}{l}\text { L. lactis subsp cremoris } \\
\text { MG1363, nisRK, pOri23 }\end{array}$ & Em & Current work \\
\hline 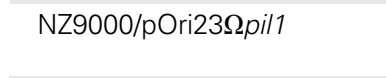 & 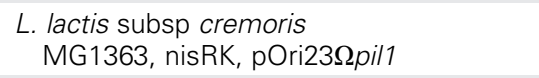 & Em & Current work \\
\hline \multicolumn{4}{|l|}{ Plasmids } \\
\hline pET-28a $(+)$ & $\begin{array}{l}\mathrm{Km} \text {, oriR } \mathrm{pBR} 322 \text {, origin } \mathrm{f1} \\
\text { promoter T7, coding sequence } \\
\text { His-Tag, terminator T7, lacZa }\end{array}$ & $\mathrm{Km}$ & $\begin{array}{l}\text { Novagen, } \\
\text { R\&D Systems }\end{array}$ \\
\hline pOri23 & $\begin{array}{l}\text { Em, oriRs pUC18 et pIP501 } \\
\text { (Gram }{ }^{-} / \text {Gram }^{+} \text {shuttle vector) }\end{array}$ & Em & [32] \\
\hline
\end{tabular}

Abbreviations: Em, Erythromycin; Km, Kanamycin; MLST, MultiLocus Sequence Typing; Tc, Tetracyclin.

for protein expression. Recombinant 6xHis-Gallo2178 and 6xHisGallo2179 were purified under native conditions by affinity chromatography on nickel-nitrilotriacetic acid columns according to the manufacturers' recommendations (Novagen). Protein purity was checked on sodium dodecyl sulfate-polyacrylamide gel electrophoresis (SDS-PAGE), and protein concentrations were determined with the BCA kit (Thermo Scientific).

\section{Generation of Rabbit and Mice Polyclonal Antibodies}

Rabbit polyclonal antibodies (pAbs) against Gallo2178 were generated by Covalab. For double-labeling experiments, pAbs against Gallo2179 were generated in mice using a very similar procedure except for the quantity of the antigen injected: $10 \mu \mathrm{g}$ per boost in each mouse, with a total of 4 boosts at 2 week-intervals.

\section{Immunoblots}

For analysis of Gallo2178 and Gallo2179 expression, cell wall proteins were boiled in Laemmli sample buffer, resolved on TrisAcetate Criterion XT gradient gels (4-12\% SDS-PAGE gels), and transferred to nitrocellulose membrane (Hybond-C; Amersham). Gallo2178 and Gallo2179 were detected using specific pAbs and horseradish peroxidase-coupled anti-rabbit or anti-mouse secondary antibodies (Zymed) and the West Pico Chemiluminescence kit (Thermo Scientific). Image capture and analysis were performed with a GeneGnome imaging system (Syngene).

\section{Immunogold Electron Microscopy}

Bacteria were grown in appropriate medium and collected after overnight growth. Scanning electron micrographs were produced as described elsewhere [28].

\section{Adherence Assay and Biofilm Formation}

Bacterial attachment and surface growth on microtiter plates were determined during growth of S. gallolyticus or recombinant L. lactis in Luria broth medium supplemented with $1 \%$ glucose and erythromycin when necessary. The 96-well polystyrene plates were coated with $0.1 \mathrm{mg} / \mathrm{mL}$ collagen I (rat tail; BD Biosciences) diluted in $1 \times$ phosphate-buffered saline overnight at $4^{\circ} \mathrm{C}$. Overnight cultures grown in Todd-Hewitt were used to inoculate Luria broth glucose medium at an optical density at $600 \mathrm{~nm}$ of 0.1 , and, after a brief vortexing, $180 \mu \mathrm{L}$ of cell suspension was dispensed into 96-well plates (Costar 3799; Corning) and incubated at $37^{\circ} \mathrm{C}$ for 2 or 24 hours. Adherent bacteria were stained with $0.1 \%$ crystal violet as described elsewhere [27]. The assay was performed in quadriplate and repeated in $\geq 3$ independent experiments.

For confocal laser-scanning microscopy (CLSM) analysis of biofilm architecture, a similar cultivation procedure was applied in 96-well polystyrene microtiter plate, which allowed for high-resolution imaging (Greiner Bio-one; microplates with a $\mu$ Clear base of $190 \pm 5 \mu \mathrm{m}$ thickness), as described elsewhere [47]. 
Table 2. Primers Used in Current Study

\begin{tabular}{|c|c|}
\hline Primers & Sequences $5^{\prime} \rightarrow 3^{\prime a}$ \\
\hline \multicolumn{2}{|l|}{ pil1 amplification } \\
\hline pilUCN34-fwd & ATTAGGGATCCAGGGAGTGATAAAGTGGTTGCT \\
\hline pilUCN34-rev & GCAATATGCATTACCGTCGCCCAAACAGTTTGA \\
\hline \multicolumn{2}{|c|}{ Recombinant protein production } \\
\hline gallo2178-Nhe1 & AGCTGCTAGCTATGATATTACTGTTGAGAACGGT \\
\hline gallo2178-BamH1 & GTTCCGGATCCTCAAGTTGAAGGAAGTGTTGC \\
\hline gallo2179-Nhe1 & GAAGTAGCTAGCGCTGATGTTAGTAATCGTGTA \\
\hline gallo2179-BamH1 & TAATGGATCCTCAACCTGTATTAGGGAATAG \\
\hline \multicolumn{2}{|c|}{ Transcriptional analyses } \\
\hline CD5 & GTGCTTTAGATAAGCGATGTTTGG \\
\hline CD6 & GCAGCCCTTACTTCTGATACTGTG \\
\hline CD42 & GATGGTGAAAAGATTGCATATACAGTC \\
\hline CD43 & ATATGTTCCACTACCACCGTTCTCAACAG \\
\hline CD44 & GACTGTTGTTATAGGCGTTAGGTTGCAAG \\
\hline CD45 & CGCTAACTGTCGGATACAATAACAAGGAC \\
\hline CD31 & GGATCCAAATGTTTACCGCAGATTACG \\
\hline CD32 & CCTGCAGTATGAGCCGTGACACTATCCGC \\
\hline gallo2177-fwd & TGACTGGTACGGGGATTATGGCTT \\
\hline gallo2177-rev & CGCTAAACCACGGTGACCTGAAAT \\
\hline gallo2178-fwd & ACTGTTGAGAACGGTGGTAGTGGA \\
\hline gallo2178-rev & GTTTGACCAGCTGTAGTGATGCCA \\
\hline gallo2179-fwd & CACTATTGAGGTCACCTGGTCGAT \\
\hline gallo2179-rev & СССАСССТGАТАСАТTTТССАТTG \\
\hline gallo16SRNA-fwd & CAGGTCTTGACATCCCGATGCTAT \\
\hline gallo16SRNA-rev & CGCTAGAGTGCCCAACTGAATGAT \\
\hline \multicolumn{2}{|c|}{ Polymerase chain reaction analyses } \\
\hline gallo2177-CD25 & TTTGACGATTAGCCTCGTCGGCATA \\
\hline gallo2177-CD26 & CGTAATCTGCGGTAAACATTTACCAC \\
\hline
\end{tabular}

a Restriction sites are in bold.

\section{Rat Model of IE}

Sterile aortic vegetations were produced in female Wistar rats by insertion of a catheter through the aortic valve, as described elsewhere [48]. The catheter was left in place throughout the experiment. Groups of animals were inoculated intravenously with $10^{3}$ or $10^{4}$ colony-forming units (CFU) for S. gallolyticus UCN34 or $10^{6} \mathrm{CFU}$ for L. lactis NZ9000/pOri23 and L. lactis NZ9000/ pOri23 $\Omega$ pil1 prepared from overnight cultures. These inoculum sizes allowed the determination of the $90 \%$ infectivity rate $\left(\mathrm{ID}_{90}\right)$ of S. gallolyticus and permit differentiation in L. lactis virulence, based on previous studies in the same model [43, 45]. Rats were euthanized 24 hours after inoculation, aortic vegetations were removed, homogenized in $1 \mathrm{~mL}$ of saline, serially diluted, and plated for colony counts. Statistic analyses were performed using the $\chi^{2}$ test, and differences were considered significant at $P<.05$.

\section{RESULTS AND DISCUSSION}

\section{Genetic Organization of the pil1 Locus in Strain UCN34}

The pil1 locus of S. gallolyticus UCN34 is composed of 3 genes encoding 2 LPXTG proteins (gallo2179 and gallo2178) and 1 sortase C (gallo2177) (Figure $1 A$ ). Such a simple genetic organization has been reported only in Actinomyces naeslundii [37]. The majority of previously characterized pilus loci in other gram-positive bacteria, such as other streptococci and enterococci, consist of 3 structural pilus genes, 1 coding for the major pilus subunit and 2 for accessory subunits acting as tip adhesin and basal anchor, and 1-3 class $C$ sortases. A transcriptional regulatory gene is often found upstream and divergently transcribed from the pilus operon. The structural proteins Gallo2178 and Gallo2179 possess the characteristic features of pilin subunits, which is a signal peptide at the $\mathrm{N}$-terminus, an LPXTG motif at its C-terminus, and a pilin motif PK in the central part. A search for conserved domains revealed the presence of structural $\mathrm{CnaB}$ domains (Pfam 05738) in both proteins. In addition, Gallo2179 contains a putative collagen-binding (COL) domain (Pfam 05737). Basic Local Alignment Search Tool (BLAST) analyses showed that Gallo2179 shares strong similarities with other collagenbinding proteins whose prototype is the Cna protein of $S$. aureus [16]. The genes surrounding this pilus gene cluster, gallo2180 and gallo2176, were annotated as genes encoding 
A

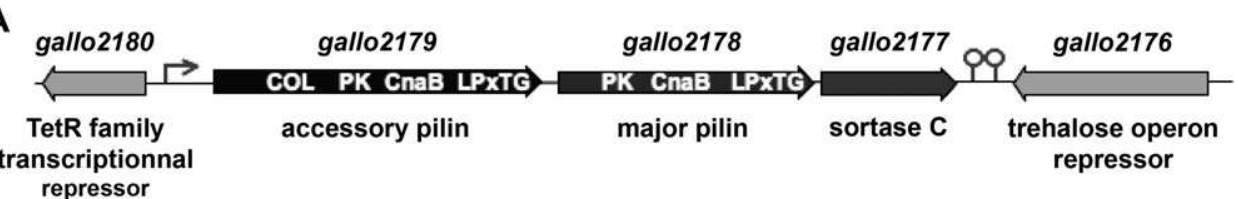

B

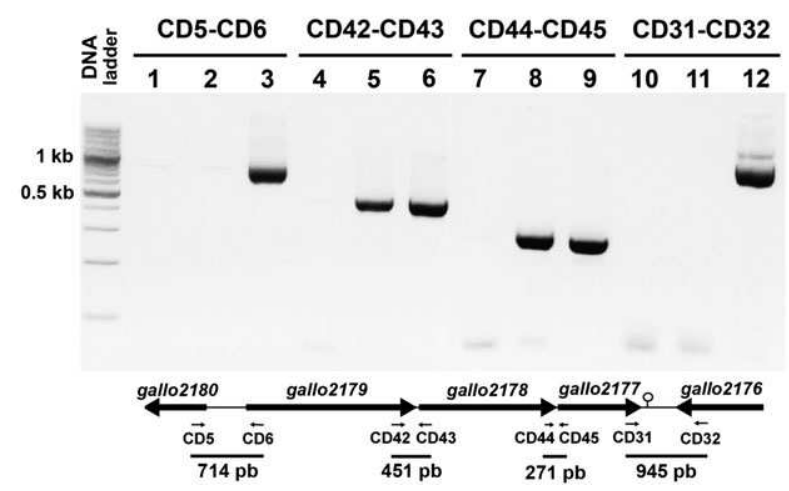

Figure 1. Physical map of pil1 operon in Streptococcus gallolyticus strain UCN34. A, Schematic representation of the pil1 locus. COL, functional collagen-binding domain (Pfam 05737); PK, pilin motif; CnaB, structural collagen-binding domain, immunoglobulinlike module (Pfam 05738); LPXTG, cell wall-anchoring domain; $r$, putative promoter; 9 , putative terminator. $B$, Determination of pil1 operon. DNA polymerase chain reaction products were amplified using the indicated oligonucleotides pairs. Lanes 1, 4, 7, and 10 represent negative controls without reverse transcription; lanes 3, 6, 9, and 12, positive controls using UCN34 chromosomal DNA; lanes 2, 5, 8, and 11, cotranscriptional analyses of gallo2179, gallo2178, and gallo2177. Thin arrows below genes indicate oligonucleotides used, and black lines represent successfully amplified fragments.

transcriptional regulators belonging to the TetR family and trehalose repressor, respectively (Figure $1 A$ ).

\section{Transcription of pil1 Locus in S. gallolyticus}

We first analyzed the transcription levels of pil1 genes in the reference strain UCN34 by quantitative reverse-transcription PCR (qRT-PCR). The 3 genes of pil1 were transcribed at similar levels, suggesting an organization in operon. Complementary RT-PCR experiments using oligonucleotides mapping the intergenic regions were realized (Figure $1 B$ ). Only 2 PCR products of 451 and 271 base pairs were obtained, showing that gallo2179, gallo2178, and gallo2177 form an operon.

In the absence of genetic tools allowing the construction of deletion mutants in S. gallolyticus, we searched in our collection for clinical isolates expressing the pill locus at levels different from those of our reference strain UCN34. Interestingly, we characterized 1 strain, NEM2470 (designated Pill ${ }^{+}$), that expresses the 3 genes of pil1 operon at a higher level (gallo2179, 5.8-fold, gallo2178, 7-fold, gallo2177, 8.5-fold) than that of UCN34. We found another strain, NEM2474 (designated Pil1 ${ }^{-}$), that does not express pil1.

\section{pil1-Encoded Proteins Gallo2178 and Gallo2179 Form Polymers of High Molecular Weight}

Pill pilus biogenesis was assessed by Western blotting of cell wall protein extracts from S. gallolyticus strains UCN34, Pil1 ${ }^{+}$, and Pill $^{-}$using specific antibodies directed against the pilus structural components Gallo2178 and Gallo2179. To unambiguously characterize this operon in isogenic strains, heterologous expression of pil1 was performed in the nonpathogenic Lactococcus lactis NZ9000 by cloning the 3 genes (gallo2179-77) into the shuttle vector pOri23 [38]. Antisera raised against both proteins are highly specific, as demonstrated by the absence of reactive protein in the extracts from S. gallolyticus $\mathrm{Pill}^{-}$and from the control strain L. lactis NZ9000/pOri23 (Figure 2A). The antiserum raised against the major pilin Gallo2178 recognized high-molecular-weight species both in S. gallolyticus UCN34 and $\mathrm{Pill}^{+}$and in the recombinant L. lactis NZ9000/pOri23 $\Omega$ pil1, with the typical laddering profile of covalently linked polymers as seen in many gram-positive bacteria [27, 33]. A similar profile of highmolecular-weight species was observed with the antiserum specific for Gallo2179 (Figure 2A). It is noteworthy that protein levels of Gallo2178 and Gallo2179 demonstrated by Western blot analysis in strains UCN34 and $\mathrm{Pill}^{+}$correlate perfectly with findings of qRT-PCR analyses.

\section{Electron Microscopy Evidence for Pilus Structure}

Immunogold electron microscopy in S. gallolyticus UCN34 and $\mathrm{Pill}^{+}$carried out with an antiserum specific for the major pilin Gallo2178 showed that each labeled bacterium possessed a few (1-4) short pilus structures (Figure $3 A$ and $3 B$ ). A strong heterogeneity in the population of $S$. gallolyticus expressing the pilin Gallo2178 was observed in both UCN34 and Pil1 ${ }^{+}$(Figure 3A), 


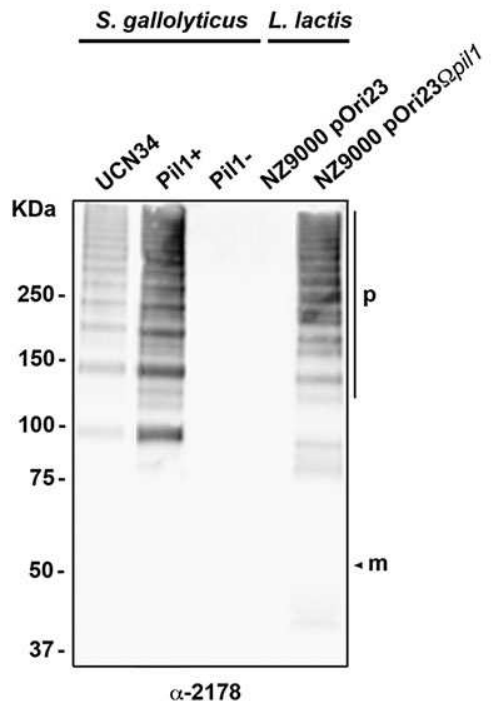

S. gallolyticus L. lactis

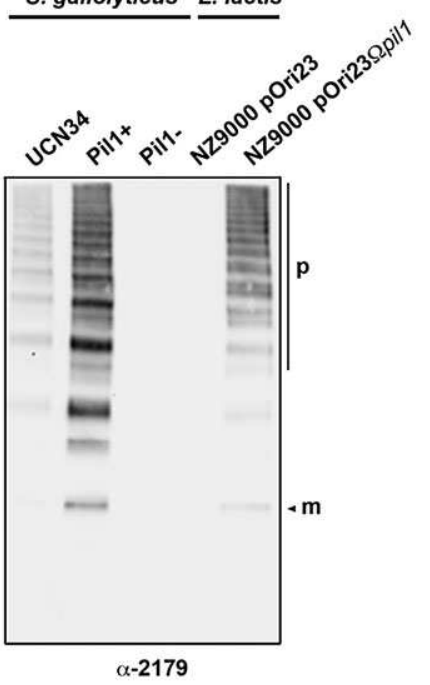

B
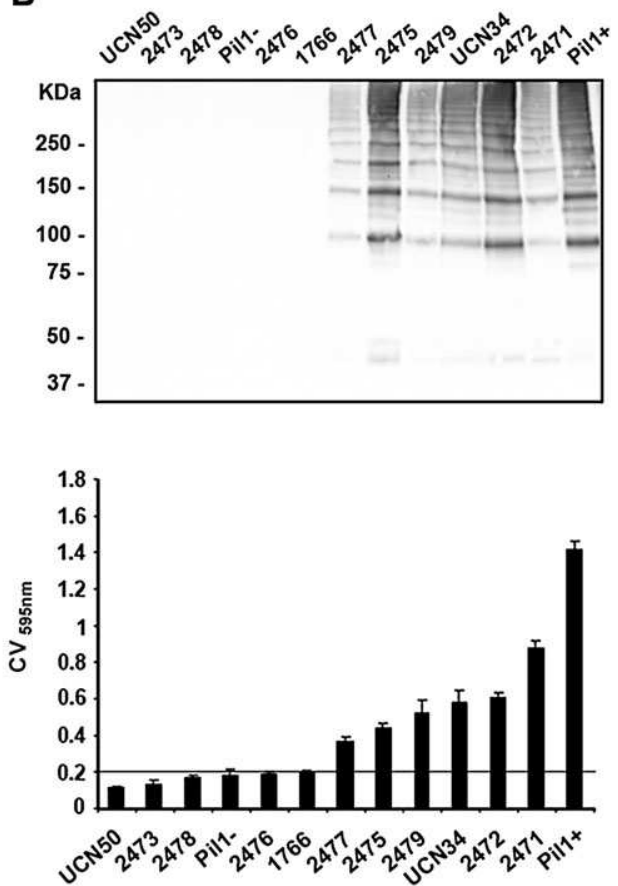

Figure 2. Pilus polymerization in Streptococcus gallolyticus wild-type and Lactococcus lactis recombinant strains. A, Western blot analysis of cell wall

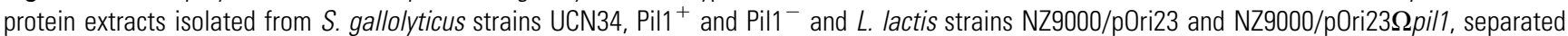
with 4\%-12\% Criterion XT sodium dodecyl sulfate-polyacrylamide gel electrophoresis and detected by means of immunoblotting using specific antiGallo2178 (left) and anti-Gallo2179 (right) polyclonal antibodies (pAbs). Equivalent amount ( 10 $\mu \mathrm{g}$ ) of total proteins was loaded in each well. Theoretical positions of Gallo2178 and Gallo2179 monomers, based on their molecular weights, are indicated by a black arrow $(\mathrm{m})$, and high-molecularweight species corresponding to pili polymers are also labeled $(p)$. $B$, Western blot analysis of cell wall protein extracts $(5 \mu \mathrm{g} /$ well) isolated from 10 additional S. gallolyticus clinical strains revealed using specific anti-Gallo2178 pAbs (top). Note adherence of $S$. gallolyticus strains to immobilized collagen I. Each well was coated with $1 \mu \mathrm{g}$ of collagen I, and $10^{7}$ bacterial colony-forming units for each strain were added. Bound bacteria were detected using crystal violet (CV) staining after extensive washing. Optical density at $595 \mathrm{~nm}\left(0 \mathrm{D}_{595 \mathrm{~nm}}\right)$ values are presented as means \pm standard deviations of 3 experiments performed in triplicate (bottom).

whereas the Pill ${ }^{-}$strain was not labeled (data not shown). L. lactis strain expressing pill was labeled with the anti-Gallo2178 antibody, and, interestingly, similar pilus structures were visualized on the bacterial cell surface (arrows, Figure 3J). The control strain L. lactis NZ9000/pOri23 was not labeled in the same experimental conditions (Figure 3I). Specific antiserum raised against the putative adhesin Gallo2179 in S. gallolyticus $\mathrm{Pill}^{+}$revealed short homopolymers of Gallo2179 (arrowheads, Figure $3 C$ and $3 D$ ). Similar structures were visualized on the surface of the recombinant $L$. lactis strain expressing pil1 (Figure $3 \mathrm{~K}$ ).

To confirm that Gallo2178 and Gallo2179 are part of the same pilus structure, we carried out a double-labeling experiment. S. gallolyticus Pil1 $^{+}$was stained with rabbit anti-Gallo2178 pAb, followed by $10 \mathrm{~nm}$ of gold-labeled immunoglobulin IgG, and then with mouse anti-Gallo2179 pAb followed by $20 \mathrm{~nm}$ of goldlabeled IgG. Typical heteropolymeric structures containing both pilin subunits, Gallo2178 constituting the core of the pilus and Gallo2179 at the tip, are shown in Figure $3 E$ (inset). However, fibers composed of Gallo2178 only (arrows, Figure 3B, 3F, and 3J), and more surprisingly of Gallo2179 only (arrowheads, Figure $3 E$ and $3 K)$, were also found. Interestingly, the collagen-binding protein Gallo2179 displays a typical pilin motif (PK) in its central part, raising the possibility of 3 types of fibers, that is, homopolymers of Gallo2179 or Gallo2178 and heteropolymers of Gallo2179-2178. To our knowledge, this structural peculiarity has never been described in other pilus-associated adhesins until now. The homologous staphylococcal and enterococcal genes, cna acm, and ace, all occur as individual genes [20]. Therefore, Gallo2179 may represent a remarkable example of evolution of an individual adhesin incorporated into a pilus fiber, thus increasing its avidity and affinity for collagen.

\section{Role of Pil1 Pilus in Primary Attachment to Collagen}

Because Gallo2179 is highly similar to other collagen-binding proteins of gram-positive bacteria, we first tested the adhesion capacity of $S$. gallolyticus UCN34 to 4 ECM proteins (collagen I and IV, fibronectin, and fibrinogen) over a 2-hour period. The reference strain UCN34 adhered preferentially to collagen I, and to a lesser extent to collagen IV (Figure 4A). Adherence to fibronectin and fibrinogen was not significant. In similar 


\section{S. gallolyticus}

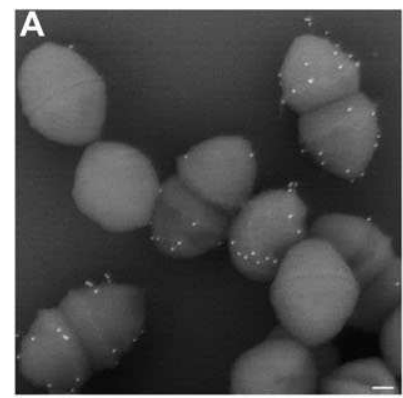

UCN34 ( $\alpha-2178)$

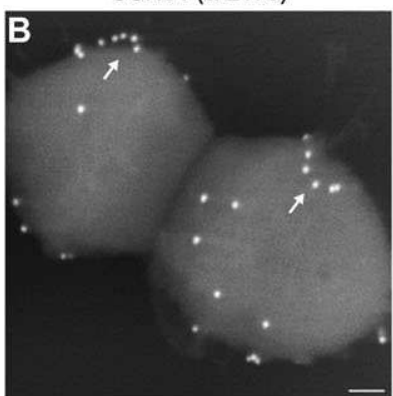

UCN34 ( $\alpha-2178)$

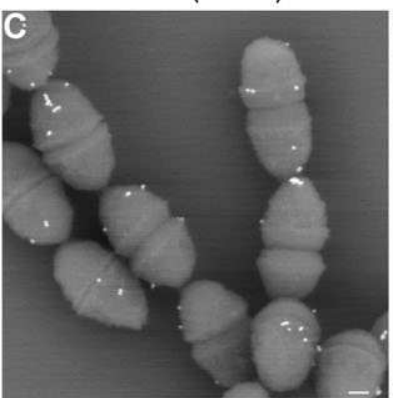

Pil1+ $(\alpha-2179)$

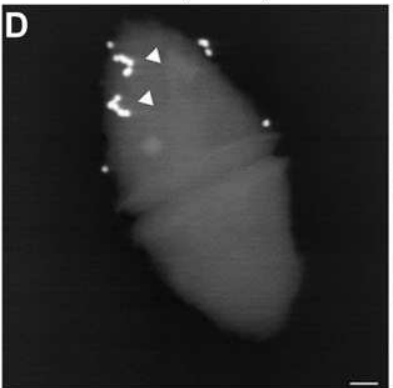

Pil1+ $(\alpha-2179)$

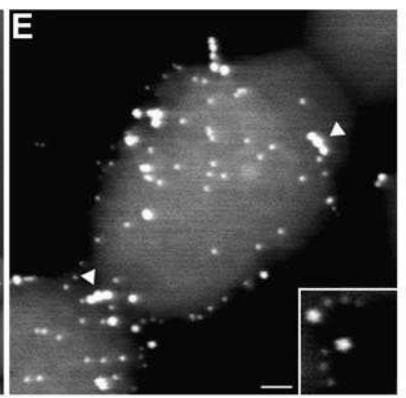

Pil1+ $(\alpha-2178, \alpha-2179)$

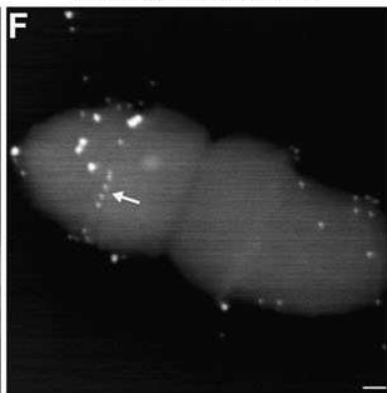

Pil1+( $\alpha-2178, \alpha-2179)$

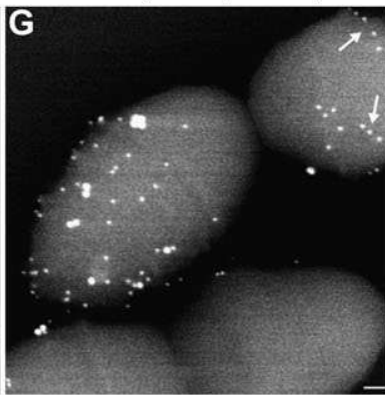

Pil1+ $(\alpha-2178, \alpha-2179)$

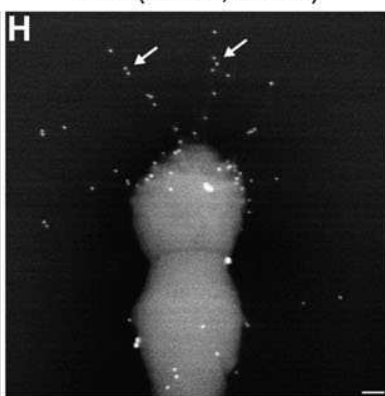

Pil1+ $(\alpha-2178, \alpha-2179)$
L. lactis

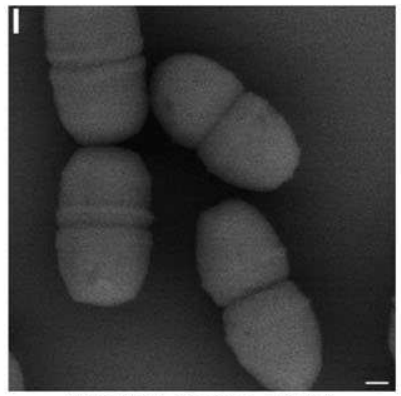

NZ9000/pOri23 ( $\alpha-2178)$

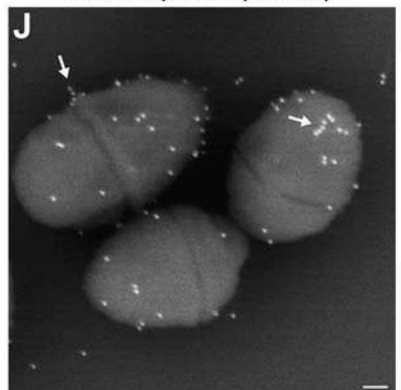

NZ9000/pOri23ßpil1 ( $\alpha-2178)$

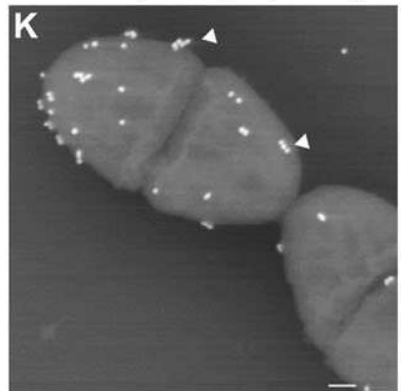

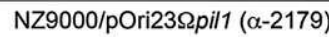

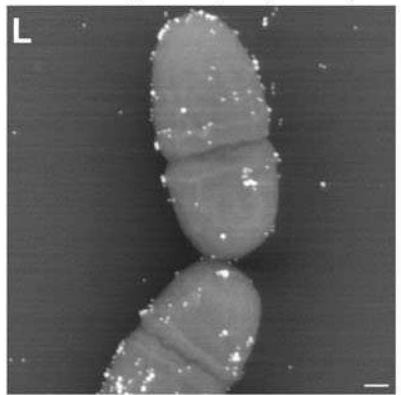

NZ9000/pOri23spil1 ( $\alpha-2178, \alpha-2179)$

Figure 3. Immunogold electron microscopy analysis of the pilus subunits Gallo2178 and Gallo2179. Streptococcus gallolyticus strains UCN34, Pil1 ${ }^{+}$ and Pil1 ${ }^{-}$and Lactococcus lactis strains NZ9000/pOri23 and NZ9000/pOri23 $\Omega$ pil1 were incubated with rabbit and mouse polyclonal antibodies raised against Gallo2178 and Gallo2179, respectively. Anti-rabbit and anti-mouse secondary antibodies were conjugated to 10- and 20-nm gold particles, respectively. E-H, For double-labeling experiments on S. gallolyticus strain Pil1 ${ }^{+}$, thin arrows indicate homopolymers of Gallo2178, whereas arrowheads in $E$ indicate Gallo2179 trimers. Inset in $E$ shows high-magnification view of 2 heteropolymers, Gallo2178-2179.

experiments, strain $\mathrm{Pill}^{+}$was found more adherent to collagen than strain UCN34, whereas Pil1 ${ }^{-}$did not adhere to any of these proteins. We also showed a dose-dependent binding of $\mathrm{Pill}^{+}$to collagen I (data not shown).

Analysis of 10 additional S. gallolyticus clinical isolates confirmed that Pill expression is necessary for adherence to collagen I
(Figure $2 B$ ). The strains that did not express Pill were unable to bind collagen, whereas those expressing Pill were adherent. However, there is not a strict quantitative correlation between Pill expression levels and collagen adhesion capacities, suggesting that other elements linked to the strain genetic background modulate this property. In addition, constitutive 
A
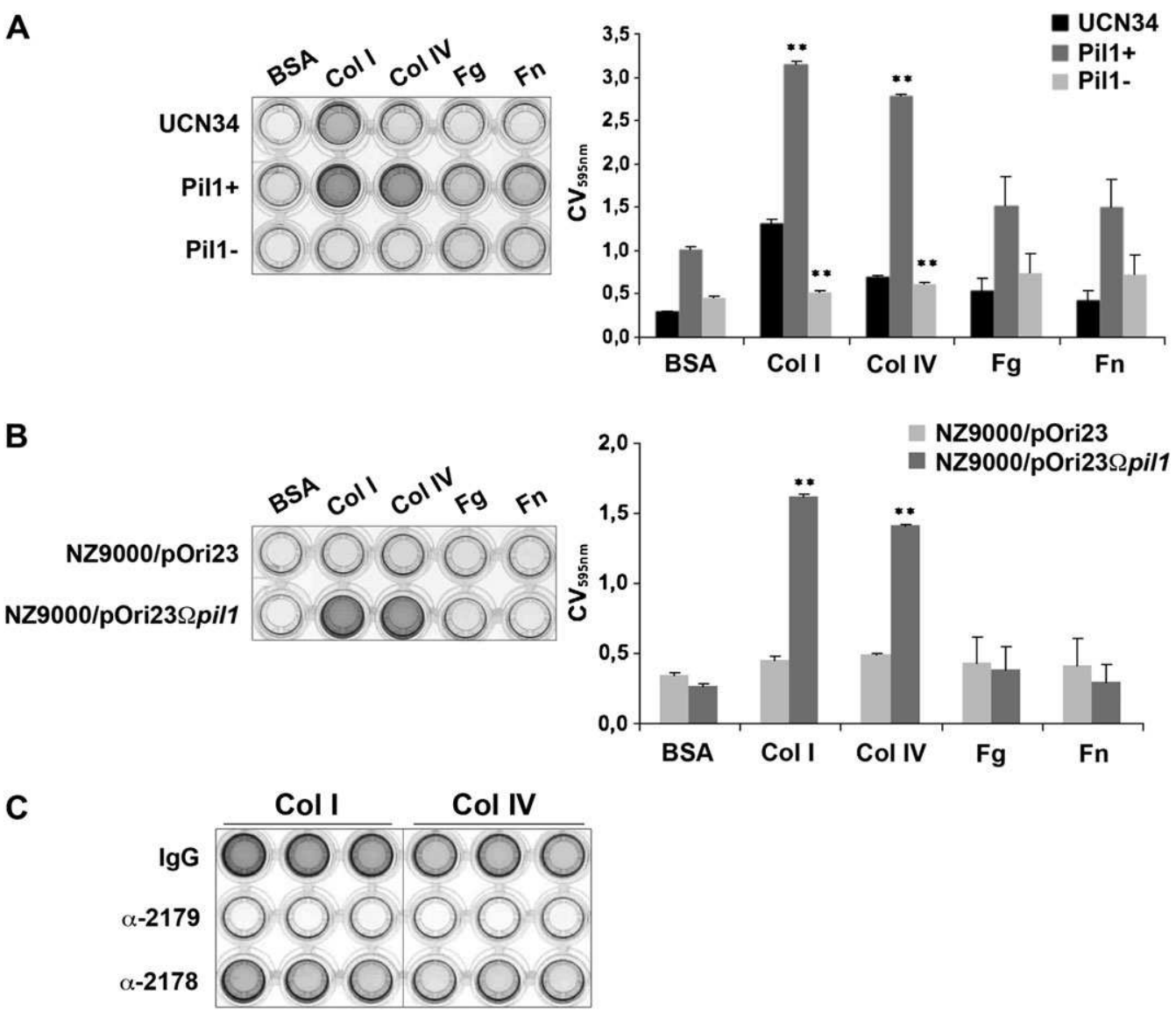

Figure 4. Adherence of Streptococcus gallolyticus wild-type strains and Lactococcus lactis recombinant strains to immobilized extracellular matrix (ECM) proteins. Microtiter wells were coated with $1 \mu \mathrm{g}$ of ECM proteins, and $10^{7}$ bacterial colony-forming units were added. The wells were washed, and bound bacteria were detected using crystal violet (CV) staining. Optical density at $595 \mathrm{~nm}\left(0 D_{595 \mathrm{~mm}}\right)$ values are presented as means \pm standard deviations for 3 experiments performed in triplicate. A, Adherence of S. gallolyticus UCN34, Pil1 ${ }^{+}$, and Pil1 ${ }^{-}$strains. B, Adherence of L. lactis NZ9000/

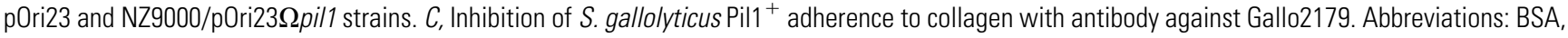
bovine serum albumin; Col I, collagen I; Col IV, collagen IV; Fg, fibrinogen; Fn, fibronectin.

expression of pill in L. lactis (NZ9000/pOri23 Rpil1) confers to this bacterium the ability to bind to collagen I and IV with a preference for collagen I, but not to fibronectin or fibrinogen. The control strain NZ9000/pOri23 did not adhere to any of the tested ECM proteins (Figure 4B).

Finally, when S. gallolyticus Pil1 ${ }^{+}$was incubated beforehand with anti-Gallo2179 pAb directed against the pill operon adhesin, bacterial adhesion to collagen was totally inhibited (Figure $4 C$ ), whereas polyclonal anti-Gallo2178 antibody showed only a slight adhesion reduction capability, potentially because of a steric hindrance. As a control, we used normal rabbit IgG (isotype control) that did not perturb bacterial adhesion. This result clearly shows that Gallo2179 is the major adhesin responsible for S. gallolyticus adhesion to collagen.

Our results are in agreement with those of a previous study showing that recombinant $\mathrm{rAcb}_{35}$ (ie, Gallo2179) produced in E. coli was able to bind collagen I, IV, and V with different affinities (I > IV > V) [20]. Collagen, the most abundant protein in human bodies and the main component of ECM, forms the only supportive fiber of cardiac valves. Of the dry weight of the human mitral valve, $67 \%$ is collagen, $74 \%$ type I, $24 \%$ type III, and $2 \%$ type V [39]. Collagen IV is a major component of the basal lamina layer underlying epithelial tissues. It is worth mentioning a study of animal isolates of S. gallolyticus that revealed no adherence to collagen I [23], which may indicate that human and animal strains possess a different repertoire of adhesins.

\section{Role of Pil1 in Biofilm Formation}

We subsequently analyzed the role of pili in biofilm formation, using 2 frequently applied experimental procedures. The first consisted of a global quantification of biofilm achieved by measuring the optical density of adhered cells with crystal violet staining [40,41]. S. gallolyticus and recombinant lactococcal strains were tested for biofilm formation on polystyrene plates coated with or without collagen I at 24 hours. For S. gallolyticus strains UCN34 and Pill ${ }^{+}$and for the L. lactis recombinant strain 


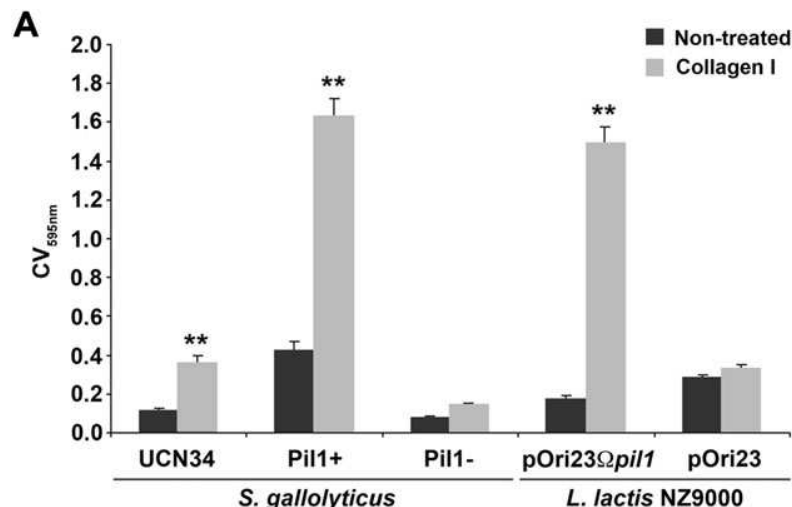

B

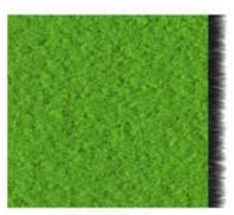

UCN34

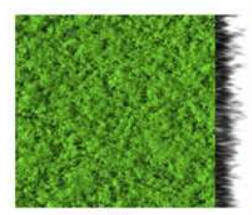

Pil1+

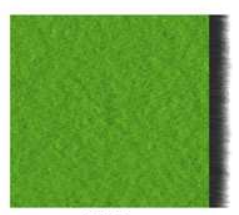

Pil1-

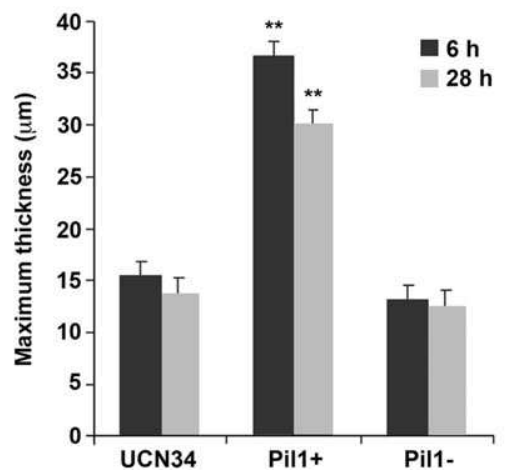

Figure 5. Role of the Pil1 pilus in biofilm formation. A, Streptococcus gallolyticus and Lactococcus lactis strains were grown in Todd-Hewitt broth at $30^{\circ} \mathrm{C}$ for 24 hours in 96-well polystyrene plates coated with $10 \mu \mathrm{g}$ of collagen I or not coated. Adherent bacteria were stained with crystal violet (CV), and quantification was performed by measuring absorbance at $595 \mathrm{~nm}$. Results are representative of 3 experiments; error bars show standard deviations. Plain bars, collagen I coating; hatched bars, not coated. $B$, top, Three-dimensional projections of biofilm architecture obtained from confocal z-stacks using IMARIS software. Images present aerial view of 28-hour biofilm structures obtained with the 3 strains of $S$. gallolyticus. Bacteria were stained in green, and shadow projection on right represents height of biofilm. Bottom, Biofilm maximal thickness (in micrometers) after 6 or 28 hours of growth (average values of 12 measurements in 4 wells). ${ }^{*} P<.01$ (1-way analysis of variance test).

NZ9000/pOri23 $\Omega$ pill, biofilm formation capacities perfectly correlated to primary attachment capacities (Figure 5A).

The second experimental procedure was carried out with a CLSM enabling direct in situ and nondestructive investigation of native multicellular structures formed by $S$. gallolyticus. Representative 28-hour biofilm structures of strains UCN34, $\mathrm{Pill}^{+}$, and Pil1 ${ }^{-}$, grown on polystyrene microplates coated with collagen I, were observed using CLSM (Figure 5B). The images corresponded to 3-dimensional reconstructions obtained from confocal stack images with IMARIS 7.0 software, including virtual shadow projection on the right (Figure $5 B$, top). Analysis of variance performed on the maximal biofilm thickness showed that Pill $^{+}$formed thicker biofilm than UCN34 and Pill ${ }^{-}$ $(P<.01)$ at both 6 and 28 hours (Figure 5B, bottom). No significant difference in biofilm thickness was found between UCN34 and Pill ${ }^{-}$, probably owing to the absence of a washing step in this procedure.

Collectively, these results suggest that $S$. gallolyticus pill operon plays a critical role in adhesion and colonization of damaged tissues exposing collagen I. Our results are in agreement with those of a very recent study showing that $S$. gallolyticus can form biofilm on collagen-rich surfaces, which in vivo are found at damaged heart valves and (pre)cancerous sites with displaced epithelium [42].

\section{Role of Pil1 in a Rat Model of Endocarditis}

We first tested the ability of $S$. gallolyticus reference strain UCN34 to infect aortic vegetations in the rat model of experimental endocarditis. The $\mathrm{ID}_{90}$ was found to be $10^{4} \mathrm{CFU}$. In rats challenged with this inoculum, 11 of 12 vegetations (92\%) were infected, whereas in those challenged with a lower inoculum of $10^{3} \mathrm{CFU}$, only 1 of $13(7 \%)$ developed infected vegetations. This $\mathrm{ID}_{90}$ value is very close to those $\left(10^{4}-10^{5} \mathrm{CFU}\right)$ of the major bacterial pathogens responsible for IE $[43,44]$.

Because the S. gallolyticus strains used in this study have different genetic backgrounds, we used the recombinant lactococcal strains, a nonpathogenic bacterium, as a surrogate organism to test the role of Pill in vivo. Rats inoculated with $10^{6} \mathrm{CFU}$ of L. lactis NZ9000/pOri23 $\Omega$ pil 1 produced infection in 9 of 11 vegetations $(82 \%)$ compared with 4 of $11(36 \%)$ with the control strain NZ9000/pOri23 $(P=.03)$ (Figure $6 A)$. These results with recombinant lactococci expressing the $S$. gallolyticus pill locus, encoding a collagen-binding protein, suggest that pill plays a critical role during the initial attachment and colonization stage of IE development. Histological analyses confirmed these results. Most animals infected with S. gallolyticus UCN34 or L. lactis NZ9000/pOri23 $2 p i l 1$ indeed displayed fibrinous valvular endocarditis associated with intralesional bacteria (Figure 6B). Furthermore, it has been shown elsewhere that recombinant lactococci expressing staphylococcal adhesins were found to increase their infectivity in experimental endocarditis [45].

\section{CONCLUSION}

This study represents the first functional characterization of a pilus locus in S. gallolyticus. The Pill pilus is made of 2 subunits (Gallo2178, the major pilin, and Gallo2179, the pilus-associated adhesin) covalently assembled by a sortase C (Gallo2177). Its involvement in the development of endocarditis identifies Pill as the first virulence factor in this intriguing pathogen. Future 


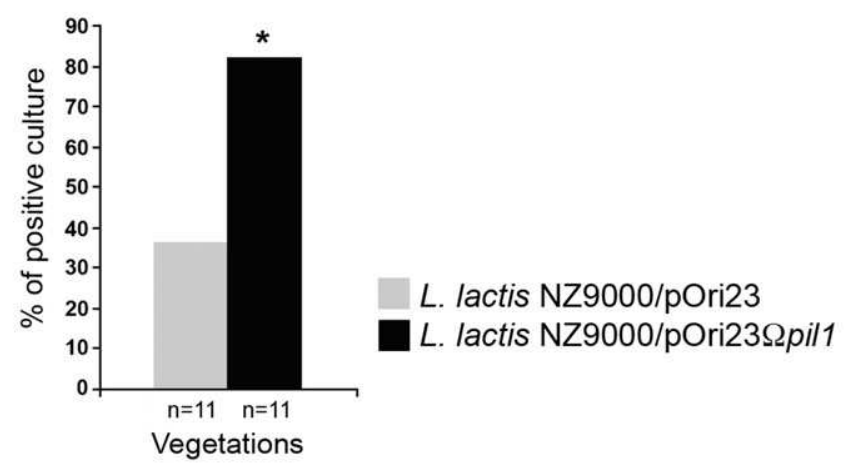

B
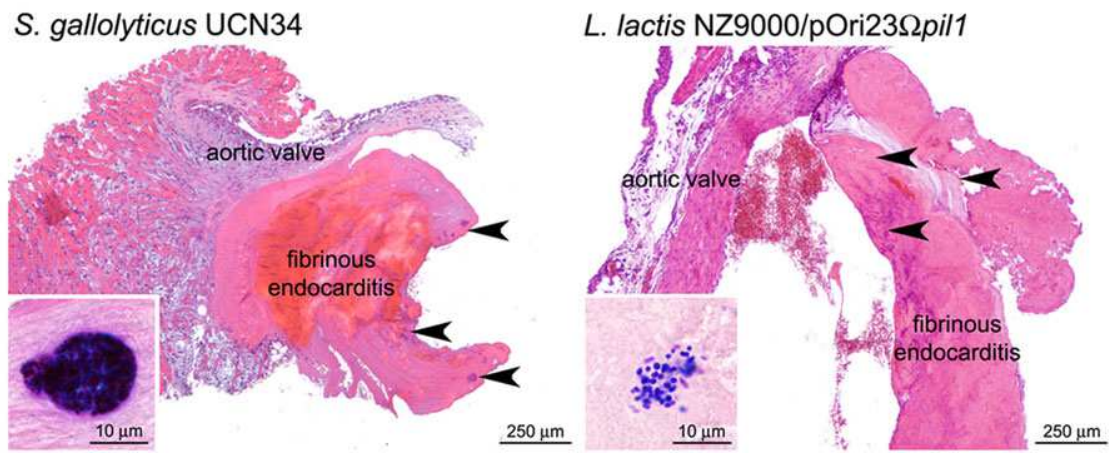

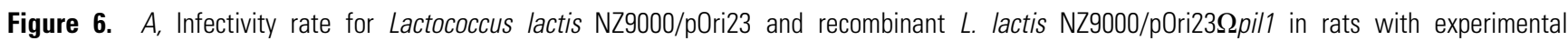
endocarditis. Groups of rats were challenged with $10^{6}$ colony-forming units of L. lactis carrying either an empty vector (p0ri23) or pil1-encoding (p0ri23 $\Omega$ pil1) plasmid. The percentage of infected vegetations was assessed after 24 hours. ${ }^{*} P=.03\left(\chi^{2}\right.$ test). $B$, Fibrinous endocarditis was observed in

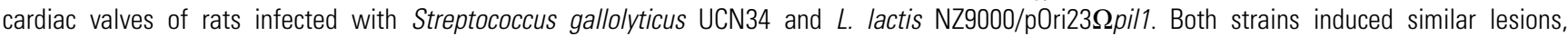
characterized by (1) endothelium ulceration, (2) accumulation of a fibrillar acidophilic material (fibrin) containing bacterial colonies (arrowheads and insets), and (3) peripheral infiltration of neutrophils and macrophages (hematoxylin-eosin staining with Gram staining in insets).

studies aim to develop genetic toolbox in S. gallolyticus, define the pilus regulation, and investigate other potential virulence factors involved in colorectal carcinoma.

\section{Supplementary Data}

Supplementary materials are available at The Journal of Infectious Diseases online (http://www.oxfordjournals.org/our_journals/jid/).

Supplementary materials consist of data provided by the author that are published to benefit the reader. The posted materials are not copyedited. The contents of all supplementary data are the sole responsibility of the authors. Questions or messages regarding errors should be addressed to the author.

\section{Notes}

Acknowledgments. We thank Samuel Bellais for help with purification of recombinant proteins $6 \mathrm{xHis}-\mathrm{Gallo} 2179$ and $6 \mathrm{xHis}-\mathrm{Gallo} 2178$ from E. coli, and Olivier Poupel for qRT-PCR experiments. We are also grateful to Marlyse Giddey and Jacques Vouillamoz for excellent technical assistance with animal experiments. We thank Claire Poyart for helpful discussion.

Financial support. This work was supported in part by the Network of Excellence EuroPathoGenomics (grant LSHB-CT-2005-512061 to P. T. C.) and the Swiss National Science Foundation (grant 310030-125325).

Potential conflicts of interest. All authors: No reported conflicts.

All authors have submitted the ICMJE Form for Disclosure of Potential Conflicts of Interest. Conflicts that the editors consider relevant to the content of the manuscript have been disclosed.

\section{References}

1. Klein RS, Recco RA, Catalano MT, Edberg SC, Casey JI, Steigbigel NH. Association of Streptococcus bovis with carcinoma of the colon. N Engl J Med 1977; 297:800-2.

2. Klein RS, Warman SW, Knackmuhs GG, Edberg SC, Steigbigel NH. Lack of association of Streptococcus bovis with noncolonic gastrointestinal carcinoma. Am J Gastroenterol 1987; 82:540-3.

3. Abdulamir AS, Hafidh RR, Mahdi LK, Al-jeboori T, Abubaker F. Investigation into the controversial association of Streptococcus gallolyticus with colorectal cancer and adenoma. BMC Cancer 2009; 9:403.

4. Boleij A, Schaeps RM, Tjalsma H. Association between Streptococcus bovis and colon cancer. J Clin Microbiol 2009; 47:516.

5. Gupta A, Madani R, Mukhtar H. Streptococcus bovis endocarditis, a silent sign for colonic tumour. Colorectal Dis 2010; 12:164-71.

6. zur Hausen H. Streptococcus bovis: causal or incidental involvement in cancer of the colon? Int J Cancer 2006; 119:xi-xii.

7. Beck M, Frodl R, Funke G. Comprehensive study of strains previously designated Streptococcus bovis consecutively isolated from human blood cultures and emended description of Streptococcus gallolyticus and Streptococcus infantarius subsp. coli. J Clin Microbiol 2008; 46:2966-72.

8. Poyart C, Quesne G, Trieu-Cuot P. Taxonomic dissection of the Streptococcus bovis group by analysis of manganese-dependent superoxide dismutase gene (sodA) sequences: reclassification of 'Streptococcus infantarius subsp. coli' as Streptococcus lutetiensis sp. nov. and of Streptococcus bovis biotype 11.2 as Streptococcus pasteurianus sp. nov. Int J Syst Evol Microbiol 2002; 52:1247-55.

9. Schlegel L, Grimont F, Ageron E, Grimont PA, Bouvet A. Reappraisal of the taxonomy of the Streptococcus bovis/Streptococcus equinus com- 
plex and related species: description of Streptococcus gallolyticus subsp. gallolyticus subsp. nov., S. gallolyticus subsp. macedonicus subsp. nov. and S. gallolyticus subsp. pasteurianus subsp. nov. Int J Syst Evol Microbiol 2003; 53:631-45.

10. Corredoira J, Alonso MP, Coira A, et al. Characteristics of Streptococcus bovis endocarditis and its differences with Streptococcus viridans endocarditis. Eur J Clin Microbiol Infect Dis 2008; 27:285-91.

11. Hoen B, Chirouze C, Cabell CH, et al. Emergence of endocarditis due to group D streptococci: findings derived from the merged database of the International Collaboration on Endocarditis. Eur J Clin Microbiol Infect Dis 2005; 24:12-6.

12. Tripodi MF, Fortunato R, Utili R, Triassi M, Zarrilli R. Molecular epidemiology of Streptococcus bovis causing endocarditis and bacteraemia in Italian patients. Clin Microbiol Infect 2005; 11:814-9.

13. De Herdt P, Haesebrouck F, Devriese LA, Ducatelle R. Biochemical and antigenic properties of Streptococcus bovis isolated from pigeons. J Clin Microbiol 1992; 30:2432-4.

14. Vanrobaeys M, De Herdt P, Charlier G, Ducatelle R, Haesebrouck F. Ultrastructure of surface components of Streptococcus gallolytics (S. bovis) strains of differing virulence isolated from pigeons. Microbiology 1999; 145:335-42.

15. Moreillon P, Que YA, Bayer AS. Pathogenesis of streptococcal and staphylococcal endocarditis. Infect Dis Clin North Am 2002; 16:297-318.

16. Zong Y, Xu Y, Liang X, et al. A 'collagen hug' model for Staphylococcus aureus CNA binding to collagen. EMBO J 2005; 24:4224-36.

17. Nallapareddy SR, Weinstock GM, Murray BE. Clinical isolates of Enterococcus faecium exhibit strain-specific collagen binding mediated by Acm, a new member of the MSCRAMM family. Mol Microbiol 2003; 47:1733-47.

18. Liu Q, Ponnuraj K, Xu Y, et al. The Enterococcus faecalis MSCRAMM ACE binds its ligand by the Collagen Hug model. J Biol Chem 2007; 282:19629-37.

19. Singh KV, Nallapareddy SR, Sillanpaa J, Murray BE. Importance of the collagen adhesin ace in pathogenesis and protection against Enterococcus faecalis experimental endocarditis. PLoS Pathog 2010; 6:e1000716.

20. Sillanpaa J, Nallapareddy SR, Qin X, et al. A collagen-binding adhesin, Acb, and ten other putative MSCRAMM and pilus family proteins of Streptococcus gallolyticus subsp. gallolyticus (Streptococcus bovis Group, biotype I). J Bacteriol 2009; 191:6643-53.

21. Angrist AA, Oka M. Pathogenesis of bacterial endocarditis. JAMA $1963 ; 183: 249-52$.

22. Sillanpaa J, Nallapareddy SR, Singh KV, Ferraro MJ, Murray BE. Adherence characteristics of endocarditis-derived Streptococcus gallolyticus ssp. gallolyticus (Streptococcus bovis biotype I) isolates to host extracellular matrix proteins. FEMS Microbiol Lett 2008; 289:104-9.

23. Vanrobaeys M, Haesebrouck F, Ducatelle R, De Herdt P. Adhesion of Streptococcus gallolyticus strains to extracellular matrix proteins. Vet Microbiol 2000; 74:273-80.

24. Vollmer T, Hinse D, Kleesiek K, Dreier J. Interactions between endocarditis-derived Streptococcus gallolyticus subsp. gallolyticus isolates and human endothelial cells. BMC Microbiol 2010; 10:78.

25. Rusniok C, Couve E, Da Cunha V, et al. Genome sequence of Streptococcus gallolyticus: insights into its adaptation to the bovine rumen and its ability to cause endocarditis. J Bacteriol 2010; 192: 2266-76.

26. Yanagawa R, Otsuki K. Some properties of the pili of Corynebacterium renale. J Bacteriol 1970; 101:1063-9.

27. Dramsi S, Caliot E, Bonne I, et al. Assembly and role of pili in group B streptococci. Mol Microbiol 2006; 60:1401-13.
28. Konto-Ghiorghi Y, Mairey E, Mallet A, et al. Dual role for pilus in adherence to epithelial cells and biofilm formation in Streptococcus agalactiae. PLoS Pathog 2009; 5:e1000422.

29. Lauer $P$, Rinaudo CD, Soriani M, et al. Genome analysis reveals pili in group B Streptococcus. Science 2005; 309:105.

30. Rosini R, Rinaudo CD, Soriani M, et al. Identification of novel genomic islands coding for antigenic pilus-like structures in Streptococcus agalactiae. Mol Microbiol 2006; 61:126-41.

31. Mandlik A, Swierczynski A, Das A, Ton-That H. Pili in gram-positive bacteria: assembly, involvement in colonization and biofilm development. Trends Microbiol 2008; 16:33-40.

32. Telford JL, Barocchi MA, Margarit I, Rappuoli R, Grandi G. Pili in gram-positive pathogens. Nat Rev Microbiol 2006; 4:509-19.

33. Ton-That H, Schneewind O. Assembly of pili on the surface of Corynebacterium diphtheriae. Mol Microbiol 2003; 50:1429-38.

34. Ton-That $\mathrm{H}$, Schneewind O. Assembly of pili in gram-positive bacteria. Trends Microbiol 2004; 12:228-34.

35. Dramsi S, Trieu-Cuot P, Bierne H. Sorting sortases: a nomenclature proposal for the various sortases of gram-positive bacteria. Res Microbiol 2005; 156:289-97.

36. Hilleringmann $M$, Ringler $P$, Muller SA, et al. Molecular architecture of Streptococcus pneumoniae TIGR4 pili. EMBO J 2009; 28:3921-30.

37. Mishra A, Das A, Cisar JO, Ton-That H. Sortase-catalyzed assembly of distinct heteromeric fimbriae in Actinomyces naeslundii. J Bacteriol 2007; 189:3156-65.

38. Que YA, Haefliger JA, Francioli P, Moreillon P. Expression of Staphylococcus aureus clumping factor A in Lactococcus lactis subsp. cremoris using a new shuttle vector. Infect Immun 2000; 68: 3516-22.

39. Cole WG, Chan D, Hickey AJ, Wilcken DE. Collagen composition of normal and myxomatous human mitral heart valves. Biochem J 1984; 219:451-60.

40. Christensen GD, Simpson WA, Younger JJ, et al. Adherence of coagulase-negative staphylococci to plastic tissue culture plates: a quantitative model for the adherence of staphylococci to medical devices. J Clin Microbiol 1985; 22:996-1006.

41. O'Toole GA, Kolter R. Initiation of biofilm formation in Pseudomonas fluorescens WCS365 proceeds via multiple, convergent signalling pathways: a genetic analysis. Mol Microbiol 1998; 28:449-61.

42. Boleij A, Muytjens CM, Bukhari SI, et al. Novel clues on the specific association of Streptococcus gallolyticus subsp gallolyticus with colorectal cancer. J Infect Dis 2011; 203:1101-9.

43. Francioli P, Moreillon P, Glauser MP. Comparison of single doses of amoxicillin or of amoxicillin-gentamicin for the prevention of endocarditis caused by Streptococcus faecalis and by viridans streptococci. J Infect Dis 1985; 152:83-9.

44. Moreillon P, Entenza JM, Francioli P, et al. Role of Staphylococcus aureus coagulase and clumping factor in pathogenesis of experimental endocarditis. Infect Immun 1995; 63:4738-43.

45. Que YA, Francois P, Haefliger JA, Entenza JM, Vaudaux P, Moreillon P. Reassessing the role of Staphylococcus aureus clumping factor and fibronectin-binding protein by expression in Lactococcus lactis. Infect Immun 2001; 69:6296-302.

46. Kuipers OP, de Ruyter PGGA, Kleerebezem M, de Vos WM. Quorum sensing-controlled gene expression in lactic acid bacteria. J Biotechnol 1998; 64:15-21.

47. Bridier A, Dubois-Brissonnet F, Boubetra A, Thomas V, Briandet R. The biofilm architecture of sixty opportunistic pathogens deciphered using a high throughput CLSM method. J Microbiol Methods 2010; 82:64-70.

48. Heraief E, Glauser MP, Freedman LR. Natural history of aortic valve endocarditis in rats. Infect Immun 1982; 37:127-31. 